\title{
Absence of rapid T cell control corresponds with delayed viral clearance in hospitalised COVID-19 patients
}

\section{Mihaela Zlei}

Leiden University Medical Center Igor A. Sidorov

Leiden University Medical Center

Simone Joosten

Leiden University Medical Center

Mirjam Heemskerk

Leiden University Medical Center https://orcid.org/0000-0001-6320-9133

Sebenzile K. Myeni

Department of Medical Microbiology, Leiden University Medical Center

\section{Cilia R. Pothast}

Leiden University Medical Center

\section{Caroline S. de Brouwer}

Leiden University Medical Center
A. Linda Boomaars-van der Zanden
Leiden University Medical Center
Kirsta E van Meijgaarden
Leiden University Medical Center

Els Wessels

Leiden University Medical Center Jacqueline J. Janse

Leiden University Medical Center Jelle Goeman

Leiden University Medical Center

\section{Christa Cobbaert}

Leiden university medical center

\section{Aloys C.M. Kroes}

Leiden University Medical Center

\section{Suzanne Cannegieter}

Leiden University Medical Center https://orcid.org/0000-0003-4707-2303

Meta Roestenberg 
Leiden University

\section{Leo Visser}

Leiden University Medical Center

\section{Marjolein Kikkert}

Leiden University Medical Center https://orcid.org/0000-0002-5779-7386

\section{Mariet Feltkamp}

Leiden University Medical center

\section{Sesmu M. Arbous}

Leiden University Medical Center

\section{Frank Staal}

Leiden University Medical Center https://orcid.org/0000-0003-1588-8519

\section{Tom Ottenhoff}

Leiden University https://orcid.org/0000-0003-3706-3403

\section{Jacques van Dongen}

Leiden University Medical Centre https://orcid.org/0000-0002-3650-7087

\section{Anna H.E. Roukens}

Leiden University Medical Center

Jutte J.C. de Vries ( $\square$ jjcdevries@lumc.nl )

Leiden University https://orcid.org/0000-0003-2530-6260

\section{Article}

Keywords: SARS-CoV-2, viral clearance, rapid T cell control

Posted Date: August 6th, 2021

DOl: https://doi.org/10.21203/rs.3.rs-783703/v1

License: (c) (i) This work is licensed under a Creative Commons Attribution 4.0 International License. Read Full License 


\section{Abstract}

SARS-CoV-2 viral load is associated with disease severity. A better understanding of immunological mechanisms involved in viral clearance is crucial to guide new therapeutic strategies.

Here, we studied the timing of viral clearance in relation to 122 immune parameters in 150 hospitalized COVID-19 patients.

Delayed viral clearance was associated with more severe disease, which occurred after the virus had been cleared in most cases. Paradoxically, delayed viral clearance was associated with over time higher maximum levels of SARS-CoV-2 specific lgG, IgA, and neutralizing antibodies, increased numbers of eosinophils, monocytes, and pro-inflammatory cyto-/chemokines. In contrast, early viral clearance and less critical illness correlated with higher levels of CD4 + and CD8 + T cells.

Collectively, our data show that absence of rapid T cell control corresponds with delayed clearance and aberrant antibody and cytokine profiles. Viral clearance often precedes critical illness, which suggests immunopathology as underlying mechanism. These data can guide treatment strategies.

\section{Introduction}

SARS-CoV-2 viral load is considered an important determinant of disease severity and mortality ${ }^{1-3}$. Viral load peaks around symptom onset and declines afterwards, with a slower rate of decline in older patients ${ }^{4,5}$. Disease severity is affected by extensive pulmonary inflammation which plays a critical role in COVID-19 pathogenesis ${ }^{6-8}$. Though related, it remains to be established to what extent virus persistence is necessary to drive ongoing tissue damage ${ }^{9}$. While the identification of correlates of protection against SARS-CoV-2 infection is a critical challenge at present, antibody responses including neutralizing titers and $\mathrm{T}$ cell responses are prime candidates based on human challenge experiments with seasonal coronavirus infections $s^{6,10}$ and recent vaccine and modelling studies ${ }^{11}$. We hypothesized that the anticipated correlates of protection from infection after vaccination would also be correlates of SARSCoV-2 clearance once infected. An extensive set of over 100 immune parameters was studied longitudinally in relation to timing to viral clearance. In contrast to our hypothesis, we observed that high maximum levels of SARS-CoV-2 neutralizing antibodies were inversely correlated with rapid viral clearance. The timing of viral clearance was analysed in relation to the peaks of the humoral and cellular responses, and all immune parameters in this study were analysed in relation to each other. Based on these analyses, we propose pathophysiological and immunological associations in terms of mechanistic correlates of protection from severe clinical disease.

\section{Results}

\section{Viral clearance preceded critical illness}


To understand the relationship between disease severity, viral clearance, and concomitant immune responses, a prospective study was performed enrolling 150 hospitalized patients with moderate to severe COVID-19. Using a longitudinal follow-up, we analysed an extensive set of virological, immunological, and clinical parameters including a daily disease severity score (see method section). Both magnitude and timing of disease severity were studied in relation to the viral clearance, defined as the time from onset of symptoms to the last positive SARS-CoV-2 PCR result $(C t<40)$. Time to viral clearance was significantly correlated with overall disease severity. Patients with delayed viral clearance, defined as viral clearance requiring $>21$ days, had significantly higher maximum daily severity scores (Fig. 1). A comparable result was found when the ratio of arterial oxygen pressure to fractional inspired oxygen $\left(\mathrm{PO}_{2} / \mathrm{FiO}_{2}\right)$ was used as discriminating parameter for pulmonary illness in ventilated patients. As anticipated, SARS-CoV-2 PCR Ct-value at day 10-14 after onset of symptoms was significantly associated with time to viral clearance, but also with fatality (Fig. 2). Subsequently, the relation of delayed viral clearance and disease severity over time was studied. In the majority of the patients, episodes of most critical illness were observed after viral clearance occurred (Fig. 1c). Since critical illness could be related to pulmonary and non-pulmonary factors, the $\mathrm{PO}_{2} / \mathrm{FiO}_{2}$ ratio was analysed here as well, with the same result: persistent pulmonary illness after viral clearance, possibly reflecting ongoing immune pathology at this stage of the disease.

\section{Paradoxical higher neutralizing antibody titers in cases with delayed clearance}

We hypothesized that anti SARS-CoV-2 specific IgG and neutralizing antibodies were correlated with viral clearance. Therefore, the kinetics and magnitude of SARS-CoV-2 IgG, IgA, and IgM responses to anti-spike $(\mathrm{S})$, anti-receptor binding domain (RBD) of spike, and anti-nucleocapsid (N) proteins were analysed in relation to viral clearance (Fig. 3). In contrast to our hypothesis, over time, the highest maximum levels of anti-N IgG, anti-S1/2 IgG, anti-RBD IgM, anti-S1 IgA, and also neutralizing antibodies were observed in patients with delayed viral clearance ( $>21$ days) and more critical illness. No differences were found between the viral clearance groups for the negative control anti-HCoV-229E IgG levels.

\section{Viral clearance dependent on (naïve) T and B cell subsets}

To study the correlation between different $T$ and $B$ cell subsets with viral clearance and disease severity, subsets of immune cells were analysed longitudinally, in correlation with the magnitude and timing of viral clearance (Fig. 4). We observed that delayed viral clearance was associated with decreased numbers of $B$ cells, post-GC B cells, switched memory B cells, and CD4-CD8-TCRgd+ cells, and there was a trend towards lower numbers of naïve $C D 4+T$ cells and naïve, pre-GC B cells in comparison with patients who cleared SARS-CoV-2 within 21 days. Patients with lower numbers of CD4+ and CD8+ T effector cells at day 10-14 post onset of symptoms had a higher risk of being admitted to ICU (Fig. 4). This suggests that both naïve and effector T and B cells are involved in early control of SARS-CoV-2. 


\section{Delayed viral clearance corresponds with significant innate cell expansion}

In contrast to the adaptive cellular responses, several innate immune cells were significantly increased in patients with delayed viral clearance: dendritic cells, monocytes (CD16+), neutrophils, and basophils, and there was a trend for eosinophils (Fig. 4). Additionally, increased neutrophil counts at day 10-14 were associated with critical illness and ICU admission. Despite these high innate cellular responses, SARSCoV-2 infection could not be controlled in these patients at an early stage.

\section{SARS-CoV-2 specific T cell fractions correlate with rapid clearance}

When analysing the proportion of SARS-CoV-2 specific CD4+ and CD8+ T cells, a significant inverse association was found with the average daily severity score: a higher proportion of SARS-CoV-2 specific CD4+ T cells was found in patients with rapid viral clearance and lower severity score (Fig 5). In contrast, a higher proportion of specific CD8+ T cells was found in patients with delayed clearance, though an effect of outlier datapoints cannot be excluded and absolute counts of specific T cells were not determined.

\section{Cytokine and chemokine 'storms' in patients with delayed viral clearance}

A large set of 83 cytokines and chemokines (Extended data Table 1) was analysed in relation to time to viral clearance (Fig. 6). Twenty-eight cyto-/chemokines correlated significantly and positively with delayed viral clearance, markers with the strongest correlations $(R>0.4)$ included: IL-4, IL-6, sIL-6Rbeta, LIF, HGF, SCGF-beta, and SCD163. The strongest correlation was observed with SCF and CXCL16 (Extended data Table 2). These cyto-/chemokines have mainly pro-inflammatory effects, indicating active inflammation. Macrophages and neutrophils are known to be among their main producing cells, corresponding with the increased number of neutrophils detected. Many of these cytokines and chemokines have been associated with chronic inflammatory conditions and extensive tissue remodelling including those related to the lung, but also with diseases associated with vascular abnormalities.

\section{Timing of clearance coincides with $B$ and $T$ cell subsets peaks}

Subsequently, the timing of viral clearance was studied in relation to the kinetics of humoral and cellular immune responses by adjustment of the time scale to the time of viral clearance (set at day 0) (Fig. 7). We reasoned that if the peak of protective immune parameters would precede or coincide with the timing 
of viral clearance, the association with viral clearance would be direct. However, the magnitude and timing of peak responses were inversely correlated with viral clearance for anti-RBD IgM and anti-S IgA levels, suggesting a lack of direct effect on viral clearance. In contrast, the timing of the peak levels of anti-N IgG, anti-S IgG, virus neutralizing antibodies, B cells, and SARS-CoV-2 specific CD4+ and CD8+ T cells correlated with the time of viral clearance, indicating a more direct role in virus control. Thus, timing of the peak (Fig.7), but not the absolute level of neutralizing antibody levels (Fig. 3) coincided with viral clearance (see above).

\section{Overall matrix of immune parameters in relation with viral clearance}

We hypothesized that immune parameters could be associated with each other due to related underlying biology, and, more specifically, that the decreased numbers of B and T cell counts were inversely correlated with higher innate cell responses and cyto-chemokine levels in the patients. This was assessed by correlating the maximum levels of all immune parameters measured for all patients in a matrix (Fig. 8a, Extended data Table 3). When focussing at comparisons between immune parameters from different sub-compartments (humoral, cellular or soluble), significant positive correlations were observed between the proportion of SARS-CoV-2 specific CD8+ T cells and IL7 and TNFSF13B, and neutralizing antibody titers and MMP-1. Additionally, matrices were designed separately for patients with delayed and rapid viral clearance (Extended data Fig. 2) and subsequently, the differences in R-values between these two groups were visualized (Fig. 8b, Extended data Table 4). Differences detected between the clearance groups with significant P-values included a negative correlation for CD4+ T cells with IL-9 and TNF-beta in the group with delayed clearance in contrast to a positive correlation with clearance $\leq 21$ days.

\section{Discussion}

To our knowledge, this is the first report focussed on the clearance of SARS-CoV-2 infection by using integrated analysis of longitudinal datasets including viral load data and $>100$ immune parameters representing a plethora of adaptive and innate components of the immune system. This approach enabled analysis of the kinetics of immune parameters in parallel, in relation to the timing of viral clearance. Similarity matrices of the studied immune parameters in relation to each other allowed the identification of parameters that differed between cases with delayed viral clearance in comparison with clearance within 21 days. Delayed viral clearance was associated with increased disease severity which is in line with other reports ${ }^{3}$, even though the highest level of critical illness usually occurred after patients had cleared SARS-CoV-2. This implies (lung) tissue-damage related immunopathology as underlying mechanism in this phase of critical illness as opposed to ongoing viral replication-related tissue damage. This underscores the importance of rapid viral control, given the apparent association of delayed viral clearance with later disease severity. Since analysis of SARS-CoV-2 loads in patients in general is largely influenced by the time points analysed ${ }^{3}$, we selected the time to viral clearance from onset of symptoms for the analyses of levels of immune parameters potentially involved. Paradoxically, delayed viral 
clearance and disease severity were associated with higher maximum levels of SARS-CoV-2 neutralizing antibodies over time. These findings are in line with other studies including patients and asymptomatic SARS-CoV-2 positive individuals, with the latter group having the lowest or even negative antibody titers, being at the other end of the spectrum of the disease ${ }^{12-16}$. It must be noted that the analysis of the magnitude and timing of the peak of responses, and seropositivity early after onset ${ }^{17}$ will give an incomplete picture when assessed as a single variable, as can be seen in our neutralizing antibody data: the peak coincides with clearance but the highest peaks are seen in cases with delayed clearance. While correlates of protection prior to viral infection in general are typically defined as cut-off levels for effective immune parameters, our findings suggest that these levels may not hold as predictor of viral clearance in infected patients. This may be explained by viral persistence as driving force for ongoing production and accumulation of antibodies, after initial failure of rapid T and B cell control, early after infection (Fig. 9).

Our antibody data were complemented by data on concomitant immunological responses and we observed a significant increase in cells and soluble mediators involved in innate immunity: eosinophils, monocytes, basophils, and the pro-inflammatory cyto-/chemokines II-4, -6, sIL-6Rbeta, LIF, HGF, and SCGFbeta were all increased in patients with delayed viral clearance and in most cases, with more severe illness. Some of these soluble components have been suggested by others as predictors of disease severity and fatal outcome ${ }^{18,19}$, in line with our data. Based on our findings, we postulate that severe COVID-19 is preceded by an inefficient virus control by adaptive ( $T$ and B cell) immunity, causing prolonged virus-induced (lung) tissue damage, which necessitates enhanced responses of the innate immune system to control and resolve the tissue damage. Clearly, such extensive innate responses are not sufficiently effective in all cases with severe COVID-19 disease courses.

In addition, our data suggest that inefficient virus control is linked to impaired levels of several $B$ and $T$ cell subsets, including CD4 + and CD8 + effector cells and naïve T and B cells, though the latter showed only a trend, possibly as a result of lower numbers of patients for this specific type of analysis. A caveat on the interpretation of these data is the fact that we only had access to peripheral blood to analyse the cellular response. It is possible that the mucosal immune responses in the upper respiratory tract and lungs were more robust in patients with early viral clearance. Another caveat of the study is the unavailability of data on viral clearance of patients that were lost to follow up within 21 days: fatal and discharged cases, which were excluded from analysis. Though the number of fatal cases excluded was relatively small (seven out of 150 patients), this may have resulted in bias towards less critical illness in the delayed clearance group and less discriminative power.

Higher maximum neutralization titers correlated with higher levels of pro-inflammatory cytokines and chemokines in our integrated analysis. In patients with delayed viral clearance, levels of activated CD $4+T$ cells and IL-9 were inversely correlated as opposed to the positive correlation in patients with earlier clearance. Future studies are needed to address a direct or indirect interaction between these two and the role of an IL-9 secreting T cell subset known as Th9 cells. 
In some reports, the increased proportion of SARS-CoV-2 specific CD $4+$ and CD8 + T cells seemed to correlate with disease severity whereas other reports do not see this correlation ${ }^{10,15,20}$. Although this proinflammatory profile may be an aggravating factor promoting immunopathogenesis, CD $4+T$ cells also have been demonstrated to control SARS, as depletion of these cells in mice resulted in delayed clearance of the virus, and more severe lung inflammation ${ }^{7,21}$. Here, we demonstrate that the proportion of SARSCoV-2 specific CD4 + T cells is higher in patients that better control the virus, and that the peak of both SARS-CoV-2 specific CD4 + and CD8 + T cells correlated with the time of viral clearance, indicating a more direct role in virus control. As argued elsewhere ${ }^{22}$, multiple high affinity antibodies are likely needed to block SARS-CoV-2 binding to ACE2 in the respiratory tract. For such antibody responses to occur, CD4 + T cell help is required to induce somatic hypermutation for increased affinity as well as class switch recombination to IgA antibodies, capable of crossing epithelial barriers. In some studies, late-responders were evaluated at a later stage after exposure, thus allowing more time to induce T cell memory ${ }^{23}$; indeed in our study the highest number of SARS-CoV-2 specific CD8 + cells were detected $>60$ days after onset of symptoms. It may be that cytotoxic responses against SARS-CoV-2 infected epithelial cells, although clearing viral infection, also lead to local lung epithelial damage contributing to disease symptoms. In contrast, CD 4 + specific T cells were inversely correlated with severity, supporting our hypothesis on the role for naïve CD4 + T cells (Fig. 9), which likely are needed to help local specific IgA and IgG3 antibody responses. In future studies, the size of the naïve TCR repertoire which correlates with the pool size of CD4 T cells would be an interesting parameter to measure in COVID-19 clinical studies. Consistently, elderly individuals, who have a smaller naïve $T$ cell pool, have decreased immune repertoires ${ }^{24}$, making it harder to quickly mount an effective adaptive immune response to a neo antigen such as SARS-CoV-2 presents.

In conclusion, our data suggest that delayed viral clearance is associated with failure of rapid $T$ and $B$ cell control, possibly due to a more narrow repertoire of naïve CD4 + T cells and B cells, followed by prolonged viral exposure causing enhanced (lung) tissue damage, requiring enhanced responses of neutralizing antibodies, chemokines, cytokines, and innate immune cells. Logically, progressive immunosenescence during aging explains why elderly are more vulnerable to infections with new microorganisms, that require control by an adaptive immune system with a highly diverse Ig and TCR repertoire. Our data suggest that correlates of protection from infection after vaccination ${ }^{11}$ might be quantitatively distinct from correlates of clearance once infected, and can guide early risk stratification of patients and treatment strategies.

\section{Methods}

Patients and sample collection

A total of 590 respiratory samples (thrice a week) and 432 serum samples (twice a week) were obtained from 150 COVID-19 patients with informed consent hospitalized in the Leiden University Medical Center (LUMC), from March 2020 to Dec 2020. The study was approved by the Medical Ethical Committee 
Leiden Delft Den Haag (NL73740.058.20) and registered in the Dutch Trial Registry (NL8589). From each patient the daily disease severity was scored. The severity score (range 0-17) includes the following parameters: respiratory rate, peripheral oxygen saturation on room air, $\mathrm{p} / \mathrm{f}$ ratio ${ }^{2}$, oxygen flow, FiO2, Glasgow coma scale score, urea, and $\mathrm{C}$ reactive protein (Extended data Table 5).

SARS-CoV-2 RT-PCR and definition of viral clearance

After extraction of nucleic acids from $200 \mu \mathrm{l}$ sample using a MagNa Pure 96 instrument (Roche Diagnostics), ten microliters extract was used for SARS-CoV-2 E-gene detection by real-time reversetranscription PCR on a CFX96 PCR instrument (Bio-Rad): $50^{\circ} \mathrm{C}$ for $5 \mathrm{~min}$, followed by $95^{\circ} \mathrm{C}$ for $20 \mathrm{~s}$ and then 45 cycles of $10695^{\circ} \mathrm{C}$ for $15 \mathrm{~s}, 55^{\circ} \mathrm{C}$ for $10 \mathrm{~s}$, and $72^{\circ} \mathrm{C}$ for $50 \mathrm{~s}$ using primers and probe described by Corman et al (2020). Viral clearance was defined as the last positive PCR available (Ct $>40)$, either $\leq 21$ or $>21$ days post onset of symptoms, which was considered delayed viral clearance ${ }^{25}$.

SARS-CoV-2 antibody assays

Semi-quantitative detection of SARS-CoV-2 anti-nucleocapsid (N) protein IgG and anti-RBD of the S protein IgM antibodies was performed on the Abbott Architect platform ${ }^{26,27}$. In this antibody chemiluminescent microparticle immunoassay (CMIA) test, the SARS-CoV-2 antigen coated paramagnetic microparticles bind to the lgG respectively IgM antibodies that attach to the viral nucleocapsid protein in human serum samples. The Sample/Calibrator index values of chemiluminescence in relative light units (RLU) of 1.40 (IgG assay) respectively 1.00 (IgM assay) and above were considered as positive per the manufacturer's instructions.

SARS-CoV-2 IgG antibody responses against the N-terminal (N-NT, position amino acids 1-246) and Cterminal (N-CT, position amino acids 181-419) antigen were analyzed in a microparticle immunoassay. $\mathrm{N}$ full-length of human coronavirus $229 \mathrm{E}$ was included as a specificity control (N-229E). In brief, as previously described previously ${ }^{28,29}$, viral gene fragments (gBlocks; IDT, San Jose, CA, USA) were cloned into pGEX-5x-3 vector (GE Healthcare Life Sciences, Chicago, IL, USA) expressed in E.coli and coupled to independent colour-coded magnetic bead sets (Bio-rad Laboratories, Hercules, CA, USA), to allow distinction between the antigens. Serum samples, 1:100 diluted, were pre-incubated with the antigen bead-mix for $1 \mathrm{hr}$ in blocking buffer and subsequently incubated with the antigen-coated bead sets for 1 hr. For detection, biotinylated goat anti-human $\lg G(H+L)$ was used, followed by streptavidin-Rphycoerythrin. The phycoerythrin signal on each bead set was analyzed in a Bioplex 200 analyzer (BioRad Laboratories, Hercules, CA, USA) and expressed as median fluorescence intensity (MFI).

Quantitative detection of SARS-CoV-2 anti-S1/S2 IgG antibodies was performed using the DiaSorin LIAISON platform. The CLIA assay consists of paramagnetic microparticles coated with distally biotinylated S1 and S2 fragments of the viral surface spike protein. RLUs proportional to the sample's anti-S1/S2 lgG levels are converted to arbitrary units (AU)/milliliter based on a standardized master curve. 
Semi-quantitative detection of SARS-CoV-2 anti-RBD IgM antibodies was performed using the Wantai IgM-ELISA (CE-IVD) kit (Sanbio) ${ }^{30}$. Briefly, the IgM u-chain capture method was used to detect IgM antibodies using a double-antigen sandwich immunoassay using mammalian cell-expressed recombinant antigens containing the RBD of the spike protein of SARS-CoV-2 as the immobilized and horseradish peroxidase-conjugated antigen. Sample/Cut-off index OD values of 1 and higher were considered positive per the manufacturer's instructions.

Semi-quantitative detection of SARS-CoV-2 anti-S1 IgA antibodies was performed using the Euroimmun $\operatorname{IgA} 2$-step ELISA ${ }^{31}$. Ratio values of 1.1 and higher were considered positive per the manufacturer's instructions.

Virus neutralization assay

Neutralization assays against live SARS-CoV-2 were performed using the microneutralization assay previously described by Algaissi and Hashem ${ }^{32}$. Vero-E6 cells [CRL-1580, American Type Culture Collection (ATCC)] were grown in Eagle's minimal essential medium (EMEM; Lonza) supplemented with $8 \%$ fetal calf serum (FCS; Bodinco BV), 1\% penicillin-streptomycin (Sigma-Aldrich, P4458) and 2 mM Lglutamine (PAA). Cells were maintained at $37^{\circ} \mathrm{C}$ in a humidified atmosphere containing $5 \% \mathrm{CO}_{2}$. Clinical isolate SARS-CoV-2/human/NLD/Leiden-0008/2020 was isolated from a nasopharyngeal sample and its characterization will be described elsewhere (manuscript in preparation). The next-generation sequencing derived sequence of this virus isolate is available under GenBank accession number MT705206.1 (https://www.ncbi.nlm.nih.gov/nuccore/1864563703) and shows one mutation in the Leiden-0008 virus spike protein compared to the Wuhan spike protein sequence resulting in Asp > Gly at position 614 (D614G) of the Spike protein. In addition, several non-silent (C12846U and C18928U) and silent mutations (C241U, C3037U, and C1448U) in other genes were found. Isolate Leiden-0008 was propagated and titrated in Vero-E6 cells using the tissue culture infective dose $50\left(\mathrm{TCID}_{50}\right)$ endpoint dilution method and the TCID 50 was calculated by the Spearman-Kärber algorithm as described ${ }^{33}$. All work with live SARS-CoV-2 was performed in a biosafety level 3 facility at Leiden University Medical Center.

Vero-E6 cells were seeded at 12,000 cells/well in 96-well tissue culture plates 1 day prior to infection. Heat-inactivated $\left(30 \mathrm{~min}\right.$ at $\left.56^{\circ} \mathrm{C}\right)$ serum samples were analysed in duplicate. The panel of sera were twofold serially diluted in duplicate, with an initial dilution of 1:10 and a final dilution of 1:1280 in $60 \mu \mathrm{L}$ EMEM medium supplemented with penicillin, streptomycin, $2 \mathrm{mM} \mathrm{L-glutamine} \mathrm{and} 2 \%$ FCS. Diluted sera were mixed with equal volumes of $120 \mathrm{TCID}_{50} / 60 \mu \mathrm{L}$ Leiden -0001 virus and incubated for $1 \mathrm{~h}$ at $37^{\circ} \mathrm{C}$. The virus-serum mixtures were then added onto Vero-E6 cell monolayers and incubated at $37^{\circ} \mathrm{C}$ in a humidified atmosphere with $5 \% \mathrm{CO}_{2}$. Cells either unexposed to the virus or mixed with $120 \mathrm{TCID}_{50} / 60 \mu \mathrm{L}$ SARS-CoV-2 were used as negative (uninfected) and positive (infected) controls, respectively. At 3 days post-infection, cells were fixed and inactivated with $40 \mu \mathrm{L} 37 \%$ formaldehyde/PBS solution/well overnight at $4^{\circ} \mathrm{C}$. The fixative was removed from cells and the clusters were stained with $50 \mu \mathrm{L} /$ well crystal violet solution, incubated for $10 \mathrm{~min}$ and rinsed with water. Dried plates were evaluated for viral cytopathic effect. Neutralization titer was calculated by dividing the number of positive wells with complete 
inhibition of the virus-induced cytopathogenic effect, by the number of replicates, and adding 2.5 to stabilize the calculated ratio. The neutralizing antibody titer was defined as the log2 reciprocal of this value. A SARS-CoV-2 back-titration was included with each assay run to confirm that the dose of the used inoculum was within the acceptable range of 30 to $300 \mathrm{TCID}_{50}$.

Circulating leukocyte counts by flow cytometry

Absolute counts of the main circulating leukocyte subsets (Extended data Fig. 3) were obtained based on an adapted standard protocol for peripheral blood sample processing for flow cytometry measurement (for detailed protocol see www.EuroFlow.org ${ }^{34}$ ) using an optimized combination of markers for surface staining (Primary Immunodeficiency Orientation Tube: PIDOT, Cytognos, Salamanca, Spain, https://www.cytognos.com/products/pidot-primary-immunodeficiency-orientation-tube ${ }^{35,36}$ ).

For a better separation of the circulating plasmablasts, CD38 was added to the PIDOT combination (Extended data Table 6 for details of antibody clones used). In summary, the procedure consisted in the bulk lysis of erythrocytes in fresh (same day) peripheral blood samples and staining of $2,5^{\star} 10^{6}$ leukocytes with reconstituted PIDOT lyophilized antibody cocktail (containing CD8 FITC, IgD FITC, CD16 PE, CD56 PE, CD4PerCPCy5.5, CD19 PeCy7, TCRgd PE-Cy7, CD3 APC, CD56 APC-C750) and drop in antibody cocktail (containing per test: $2 \mu \mathrm{L}$ CD27 BV421, 2,5 $\mu \mathrm{L}$ CD45RA BV510, $2 \mu \mathrm{L}$ CD38 BV605, 0,6 $\mu \mathrm{L}$ pure CD38) (Extended data Table 6) in a final $100 \mu \mathrm{L}$ staining volume. The data (at least 1 million events) were acquired on a 3-laser Cytek®Aurora instrument (Cytek Biosciences, Fremont, CA, USA) at the Flow cytometry Core Facility (FCF) of Leiden University Medical Center (LUMC) in Leiden, Netherlands (https://www.lumc.nl/research/facilities/fcf). For data analysis, the Infinicyt software (Cytognos SL, Salamanca, Spain) was used. The cell subtypes identified and their expression profiles reflecting the gating strategy used are presented in Extended data Fig. 3. The absolute counts per $\mu \mathrm{L}$ fresh blood were determined by a double platform approach, using the absolute fresh leukocyte counts determined prior sample processing with hematological analyzer (Sysmex) to the Statistics Configure tool of the Infinicyt software.

SARS-CoV-2 specific T cells

Blood was collected in CPT tubes (BD Biosciences, cat\#362753) from which PBMCs were isolated using Ficoll-Isopaque. After cryopreservation, $1 \times 10^{6}$ PBMCs were cultured in IMDM (Lonza, cat\#BE12-722F) supplemented with 10\% FCS (Sigma Aldrich, cat\#F7524), 1.4\% L-glutamine (Lonza, cat\#BE17-605E), and $1 \%$ Pen/Strep (Lonza, cat\#DE17-602E). T cells were stimulated with $1 \mu \mathrm{g} / \mathrm{mL}$ SARS-CoV-2 peptide pool covering nucleocapsid (Miltenyi, cat\#130-126-699), membrane (Miltenyi, cat\#130-126-703), and immunodominant regions of the spike protein (Miltenyi, cat\#130-126-701). 1\% DMSO (Merck, cat\#1029311000) or $1 \mu \mathrm{g} / \mathrm{mL} \mathrm{CMV} \mathrm{pp65} \mathrm{peptide} \mathrm{pool} \mathrm{were} \mathrm{used} \mathrm{as} \mathrm{negative} \mathrm{and} \mathrm{positive} \mathrm{control,}$ respectively. After one hour incubation, $5 \mu \mathrm{g} / \mathrm{mL}$ Brefeldin A (Sigma Aldrich) was added and after 16-hour incubation, the PBMCs were stained using Zombie-Red (biolegend, cat\#423110), CD4-Pe-Cy7 (Beckman Coulter, cat\#737660), and CD8-APC-H7 (BD Biosciences, cat\#560179) for 30 minutes at $4^{\circ} \mathrm{C}$. This step 
was followed by an 8 minute $1 \%$ paraformaldehyde fixation at room temperature and subsequent permeabilization with $0.1 \%$ saponin (Sigma Aldrich) for 20 minutes at $4^{\circ} \mathrm{C}$. Intracellular staining was performed with an antibody mix containing the following antibodies: CD137-APC (BD Pharmingen, cat\#550890), CD154-Pacific Blue (Biolegend, cat\#310820), and IFNY-BV711 (BD Biosciences, cat\#564039) in $0.1 \%$ saponin for 30 minutes at $4^{\circ} \mathrm{C}$. After staining, the cells were resuspended in $0.1 \%$ saponin and measured on a 5-laser Cytek Aurora. The analysis was performed using FlowJo V10.7.1. (BD Biosiences), in short: The lymphocytes were gated based on FSC-A and FSC-H. Zombie-Red negative cells were considered alive and T cell subsets were defined by expression of CD4 or CD8. Finally, activated CD $4+$ and CD $8+T$ cells were gated based on CD154 + CD137 + of total CD $4+$ and CD137 + IFNy + of total CD8 + T cells. SARS-CoV-2 CD 4 + or CD8 + frequencies were calculated by subtracting the background (DMSO) and taking the sum of the three stimulations (=(activated in S\% - activated in DMSO\%) + (activated in M\% - activated in DMSO\%) + (activated in N\% - activated in DMSO\%)).

Cytokine/chemokine measurements

Cytokines and chemokines were measured in serum by bead based multiplex assays using the BioPLex 100 system for acquisition as previously described ${ }^{37}$. Standard curves were provided with kits and a pooled sample of 4 Covid-19 patients was included as internal reference in all assays. Four commercially available kits were used Bio-Plex Pro ${ }^{\mathrm{TM}}$ Human Cytokine Screening Panel 48-plex; Bio-Plex Pro ${ }^{\text {tm }}$ Human Chemokine Panel 40-Plex; Bio-Plex Protm Human Inflammation Panel 1, 37-

Plex; Bio-Plex Pro ${ }^{\text {tm }}$ Human Th17 panel (IL-17F, IL-21, IL-23, IL-25, IL-31, IL-33) (all from Bio-Rad, Veenendaal, The Netherlands).

Statistical analysis

Median with inter-quartiles range (IQR) were used to report continuous variables visualized in violine plots. Groups (early versus late clearance, non-ICU versus ICU) were compared with non-parametric MannWhitney $U$ test. Correlations were analysed using Pearson's correlation coefficient. Imputation for missing data (death or transfer within 21 days) was not performed, cases discharged to home within 21 days were considered early clearance and included in rank correlation analyses. Tests with $p$-values $\leq 0.05$ were considered statistically significant. Hierarchical clustering of correlation values were produced for all immune parameters (maximum level per patient) in relation to each other, and for the groups early versus delayed viral clearance. Multiple testing correction of confidence values was done using false discovery rate (FDR, Benjamini-Hochberg correction ${ }^{38}$ ). Statistical analysis were performed using statistical libraries of R (Pearson correlation coefficients, heatmaps with hierarchical clustering, violine plots) and Perl (Mann-Whitney U test).

\section{Declarations}

Acknowledgements 
This work is supported by crowd funding Wake Up To Corona, organised by the Leiden University Fund. The authors gratefully acknowledge the Flow cytometry Core Facility (FCF) of Leiden University Medical Center (LUMC) in Leiden, the Netherlands (https://www.lumc.nl/research/facilities/fcf), coordinated by dr. K. Schepers and M. Hameetman, run by the FCF Operators E.F.E de Haas, J.P. Jansen, D.M. Lowie, S. van de Pas, and G.IJ. Reyneveld (Directors: Prof. F.J.T. Staal and Prof. J.J.M. van Dongen) for technical and experimental support, cell sorting assistance, intellectual discussions, and data analysis support.

\section{Competing interests}

The authors declare no competing interests

\section{References}

1. Fajnzylber, J., et al. SARS-CoV-2 viral load is associated with increased disease severity and mortality. Nat Commun 11, 5493 (2020).

2. Westblade, L.F., et al. SARS-CoV-2 Viral Load Predicts Mortality in Patients with and without Cancer Who Are Hospitalized with COVID-19. Cancer Cell 38, 661-671 e662 (2020).

3. Zheng, S., et al. Viral load dynamics and disease severity in patients infected with SARS-CoV-2 in Zhejiang province, China, January-March 2020: retrospective cohort study. BMJ 369, m1443 (2020).

4. He, X., et al. Temporal dynamics in viral shedding and transmissibility of COVID-19. Nat Med 26, 672-675 (2020).

5. Neant, N., et al. Modeling SARS-CoV-2 viral kinetics and association with mortality in hospitalized patients from the French COVID cohort. Proc Natl Acad Sci U S A 118(2021).

6. Huang, A.T., et al. A systematic review of antibody mediated immunity to coronaviruses: kinetics, correlates of protection, and association with severity. Nat Commun 11, 4704 (2020).

7. Tay, M.Z., Poh, C.M., Renia, L., MacAry, P.A. \& Ng, L.F.P. The trinity of COVID-19: immunity, inflammation and intervention. Nat Rev Immuno/ 20, 363-374 (2020).

8. Yang, L., et al. COVID-19: immunopathogenesis and Immunotherapeutics. Signal Transduct Target Ther 5, 128 (2020).

9. Schurink, B., et al. Viral presence and immunopathology in patients with lethal COVID-19: a prospective autopsy cohort study. Lancet Microbe 1, e290-e299 (2020).

10. Le Bert, N., et al. SARS-CoV-2-specific T cell immunity in cases of COVID-19 and SARS, and uninfected controls. Nature 584, 457-462 (2020).

11. Khoury, D.S., et al. Neutralizing antibody levels are highly predictive of immune protection from symptomatic SARS-CoV-2 infection. Nat Med (2021).

12. Seow, J., et al. Longitudinal observation and decline of neutralizing antibody responses in the three months following SARS-CoV-2 infection in humans. Nat Microbio/ 5, 1598-1607 (2020). 
13. Legros, V., et al. A longitudinal study of SARS-CoV-2-infected patients reveals a high correlation between neutralizing antibodies and COVID-19 severity. Cell Mol Immunol 18, 318-327 (2021).

14. Jeewandara, C., et al. SARS-CoV-2 neutralizing antibodies in patients with varying severity of acute COVID-19 illness. Sci Rep 11, 2062 (2021).

15. Poland, G.A., Ovsyannikova, I.G. \& Kennedy, R.B. SARS-CoV-2 immunity: review and applications to phase 3 vaccine candidates. Lancet 396, 1595-1606 (2020).

16. Tan, A.T., et al. Early induction of functional SARS-CoV-2-specific T cells associates with rapid viral clearance and mild disease in COVID-19 patients. Cell Rep 34, 108728 (2021).

17. Dispinseri, S., et al. Neutralizing antibody responses to SARS-CoV-2 in symptomatic COVID-19 is persistent and critical for survival. Nat Commun 12, 2670 (2021).

18. Del Valle, D.M., et al. An inflammatory cytokine signature predicts COVID-19 severity and survival. Nat Med 26, 1636-1643 (2020).

19. Angioni, R., et al. Age-severity matched cytokine profiling reveals specific signatures in Covid-19 patients. Cell Death Dis 11, 957 (2020).

20. Grifoni, A., et al. Targets of T Cell Responses to SARS-CoV-2 Coronavirus in Humans with COVID-19 Disease and Unexposed Individuals. Cell 181, 1489-1501 e1415 (2020).

21. Chen, J., et al. Cellular immune responses to severe acute respiratory syndrome coronavirus (SARSCoV) infection in senescent BALB/c mice: CD $4+T$ cells are important in control of SARS-CoV infection. J Viro/ 84, 1289-1301 (2010).

22. Khatri, I., Staal, F.J.T. \& van Dongen, J.J.M. Blocking of the High-Affinity Interaction-Synapse Between SARS-CoV-2 Spike and Human ACE2 Proteins Likely Requires Multiple High-Affinity Antibodies: An Immune Perspective. Front Immunol 11, 570018 (2020).

23. Thieme, C.J., et al. Robust T Cell Response Toward Spike, Membrane, and Nucleocapsid SARS-CoV-2 Proteins Is Not Associated with Recovery in Critical COVID-19 Patients. Cell Rep Med 1, 100092 (2020).

24. Britanova, O.V., et al. Age-related decrease in TCR repertoire diversity measured with deep and normalized sequence profiling. J Immuno/ 192, 2689-2698 (2014).

25. Xue, J., et al. Risk factors for prolonged viral clearance in adult patients with COVID-19 in Beijing, China: A prospective observational study. Int Immunopharmacol 89, 107031 (2020).

26. Escribano, P., et al. Detection of SARS-CoV-2 antibodies is insufficient for the diagnosis of active or cured COVID-19. Sci Rep 10, 19893 (2020).

27. Maine, G.N., et al. Longitudinal characterization of the IgM and IgG humoral response in symptomatic COVID-19 patients using the Abbott Architect. J Clin Viro/ 133, 104663 (2020).

28. Kamminga, S., et al. Development and Evaluation of a Broad Bead-Based Multiplex Immunoassay To Measure IgG Seroreactivity against Human Polyomaviruses. J Clin Microbio/ 56(2018).

29. van der Meijden, E., et al. Seroprevalence of trichodysplasia spinulosa-associated polyomavirus. Emerg Infect Dis 17, 1355-1363 (2011). 
30. Zhao, J., et al. Antibody Responses to SARS-CoV-2 in Patients With Novel Coronavirus Disease 2019. Clin Infect Dis 71, 2027-2034 (2020).

31. Beavis, K.G., et al. Evaluation of the EUROIMMUN Anti-SARS-CoV-2 ELISA Assay for detection of IgA and IgG antibodies. J Clin Virol 129, 104468 (2020).

32. Algaissi, A. \& Hashem, A.M. Evaluation of MERS-CoV Neutralizing Antibodies in Sera Using Live Virus Microneutralization Assay. Methods Mol Bio/ 2099, 107-116 (2020).

33. Killington, J.C.H.a.R.A. Virus Isolation and Quantitation. in Virology Methods Manual 1st edition 2546 (Academic Press, 1996).

34. van der Velden, V.H.J., et al. Optimization and testing of dried antibody tube: The EuroFlow LST and PIDOT tubes as examples. J Immunol Methods 475, 112287 (2019).

35. Van Dongen, J.J.M., et al. EuroFlow-Based Flowcytometric Diagnostic Screening and Classification of Primary Immunodeficiencies of the Lymphoid System. Front. Immunol. 10, 1271 (2019).

36. Van der Burg, M., et al. The EuroFlow PID Orientation Tube for Flow Cytometric Diagnostic Screening of Primary Immunodeficiencies of the Lymphoid System. Front. Immunol. 10, 246 (2019).

37. van Meijgaarden, K.E., et al. Cross-laboratory evaluation of multiplex bead assays including independent common reference standards for immunological monitoring of observational and interventional human studies. PLoS One 13, e0201205 (2018).

38. Benjamini Y., H.Y. Controlling the false discovery rate: a practical and powerful approach to multiple testing. Journal of the Royal Statistical Society 57, 289-300 (1995).

\section{Figures}



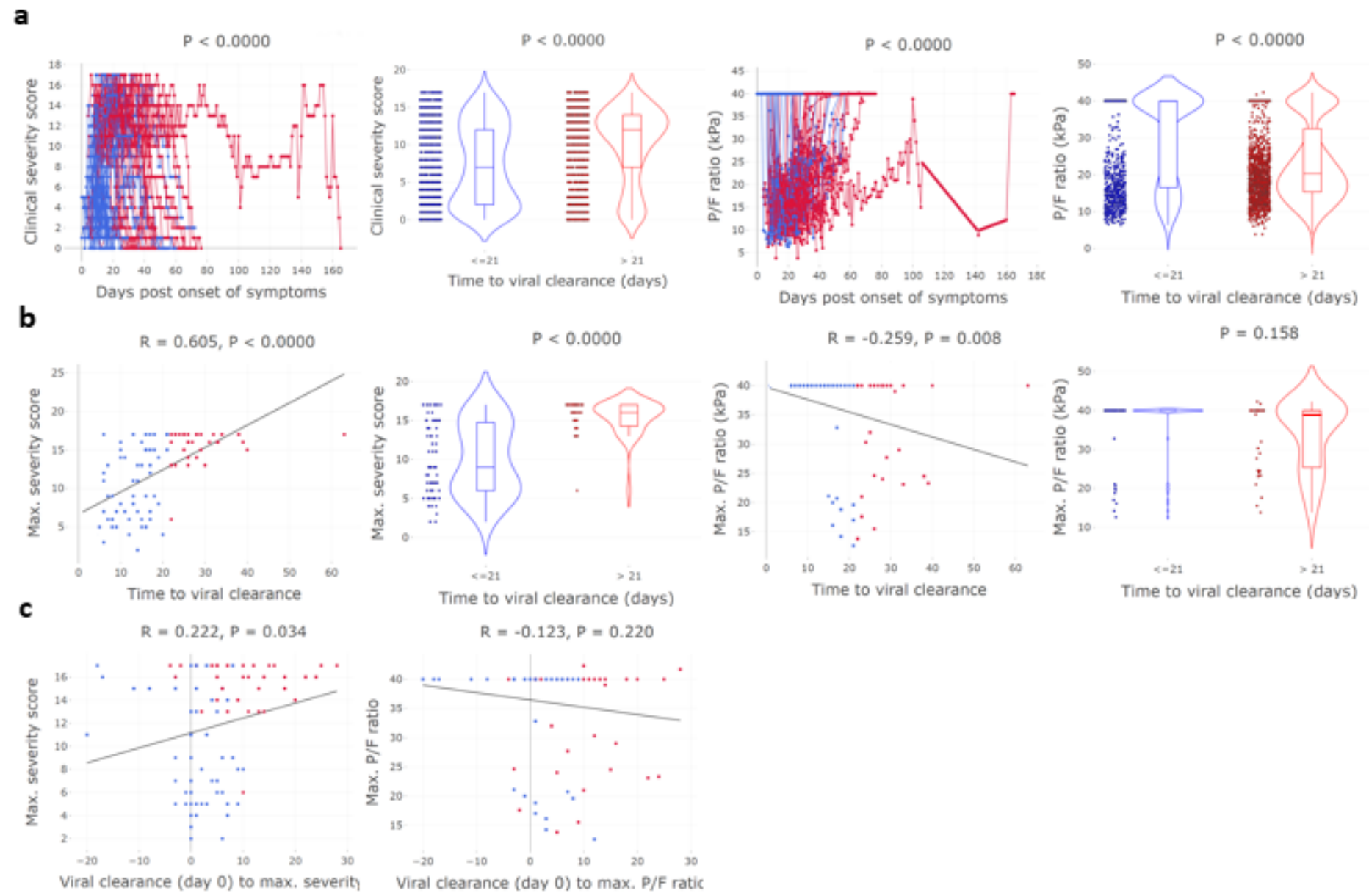

\section{Figure 1}

Clinical parameters in relation to viral clearance. a. Daily severity scores and P02/FiO2 (P/F) ratios: kinetics (in relation to the time post onset of symptoms, per patient) and median values (per groups with rapid: $\leq 21$ days or delayed: $>21$ days viral clearance); $39.9 \mathrm{kPa}$ stands for the minimum disease severity. b. The maximum (max.) daily severity score and $\mathrm{P} / \mathrm{F}$ ratio per patient in relation to time to viral clearance, defined as the last positive PCR. c. Number of days between viral clearance (vertical line, day 0 ) in relation to the maximum severity scores and to $P / F$ ratio, respectively; viral clearance $\leq 21$ days (blue lines/ dots)/ > 21 days (red lines/ dot); N=102. 

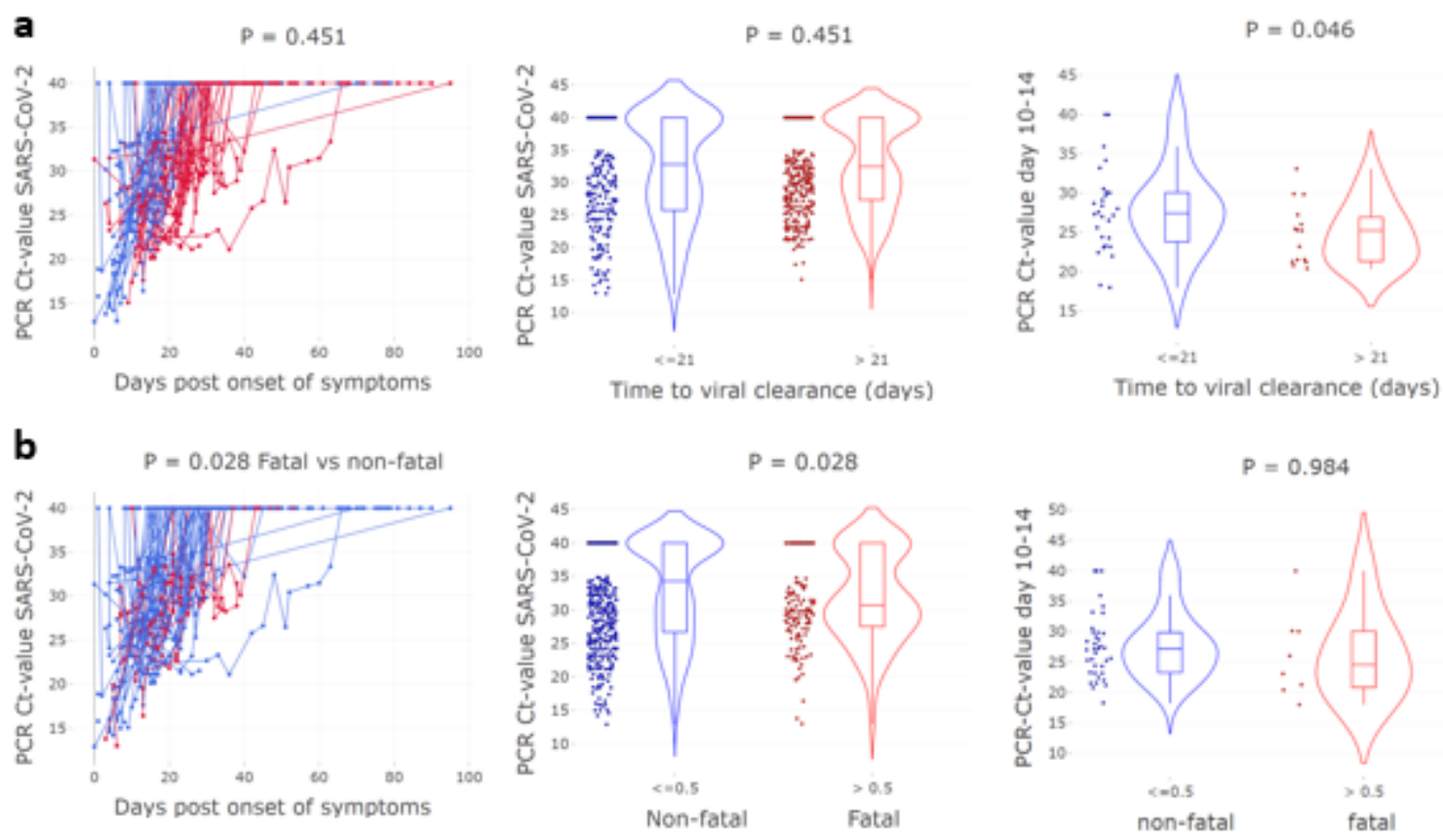

Figure 2

PCR SARS-CoV-2 Ct-values in relation to time to viral clearance. a. kinetics in relation to the time post onset of symptoms, per patient (left), all data (middle) and data at day 10-14 data (right) grouped according to the time to viral clearance $\leq 21$ days (blue) $/>21$ days (red). b. kinetics in relation to the time post onset of symptoms, per patient (left), all data (middle) and data at day 10-14 (right) grouped according to the outcome: fatal (red)/ non-fatal cases (blue); $N=111$. 

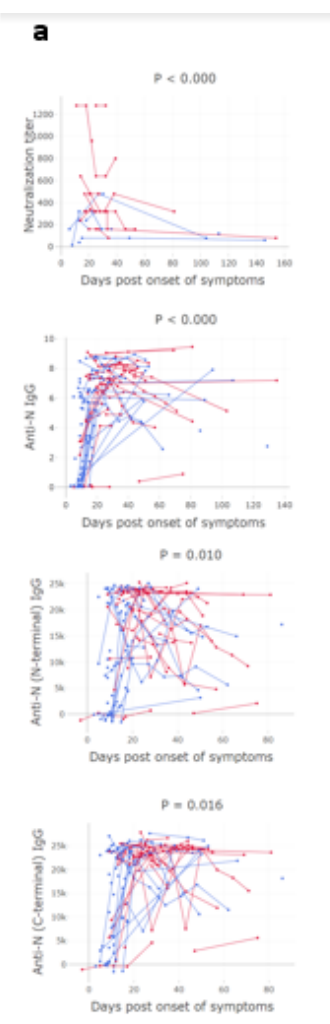

$P=0.270$
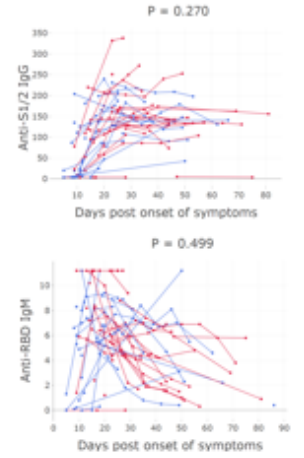

$p=0.596$

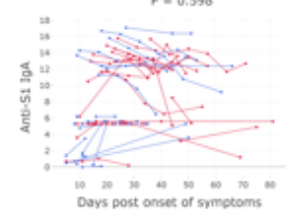

$p=0.576$

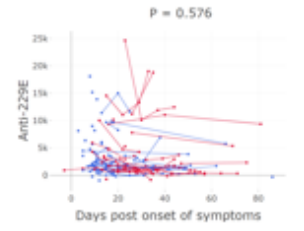

\section{b}
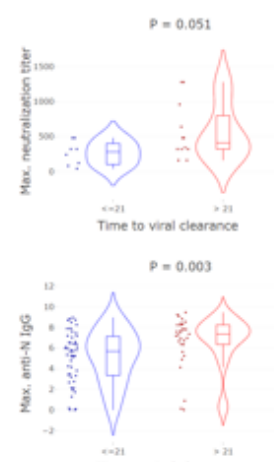

Time to viral clearance

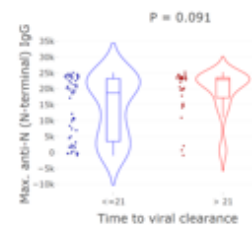

c
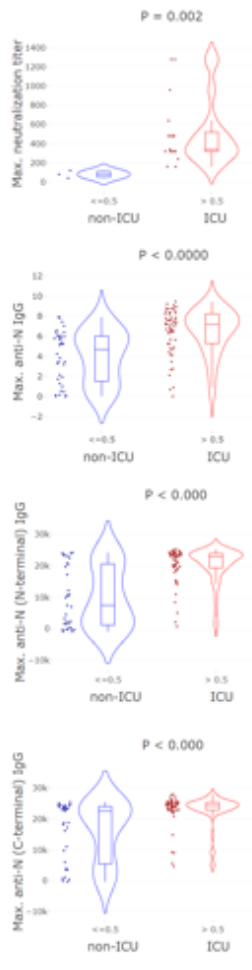

Time to viral clearance

$p=0.181$
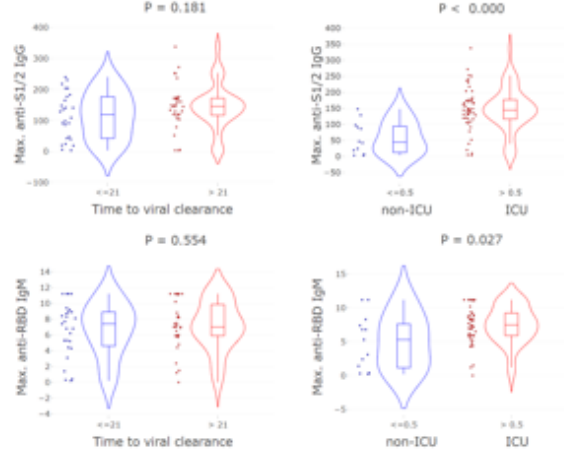

$P=0.319$

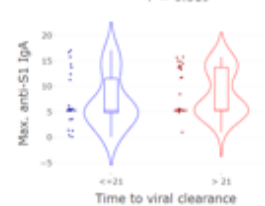

$p=0.522$

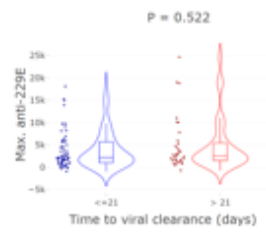

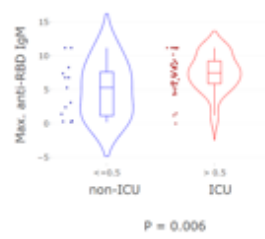
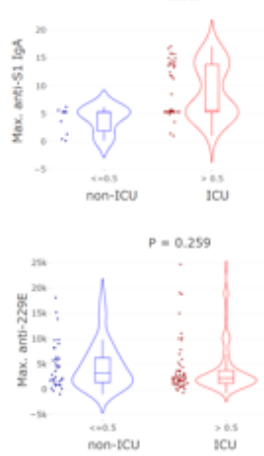

\section{Figure 3}

SARS-CoV-2 specific (neutralizing) antibody responses in hospitalized patients in relation to the day of viral clearance, with anti-HCOV-229E IgG as control. a. kinetics of SARS-CoV-2 specific (neutralizing) antibody titers in relation to the time post onset of symptoms, per patient. b. maximum values grouped by the time to viral clearance: $\leq 21$ (blue)/ > 21 (red) days; $N=95$ patients, 208 samples. c. Maximum values grouped by non-ICU (blue)/ ICU (red) patients. 

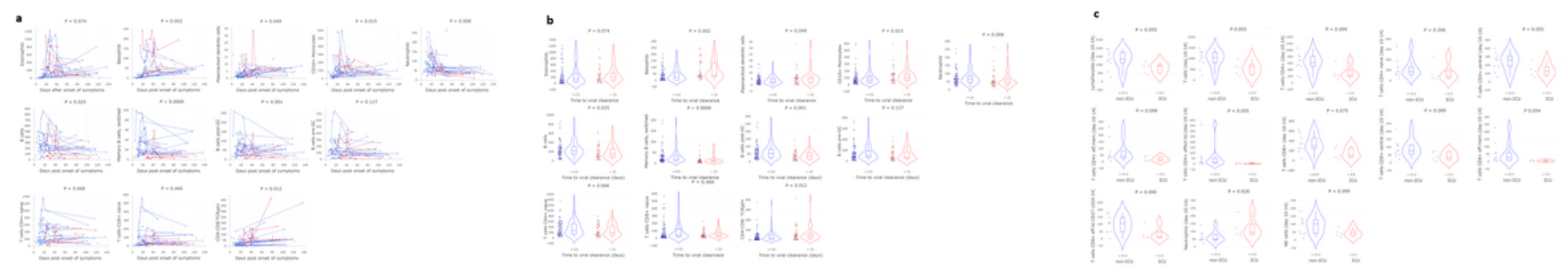

\section{Figure 4}

Absolute counts of circulating leukocyte subsets in relation to the time to viral clearance, a selection of subsets based on P-values (see Extended data Fig. 1). a. kinetics of circulating leukocyte subsets (measured as cells/ $\mu \mathrm{L}$ ) in relation to the time post onset of symptoms, per patient. b. all data grouped by the time to viral clearance: $\leq 21$ (blue)/ $>21$ (red) days; $\mathrm{N}=45$ patients, 133 samples. c. Mean values at day 10-14 grouped by non-ICU (blue)/ ICU (red) patients.
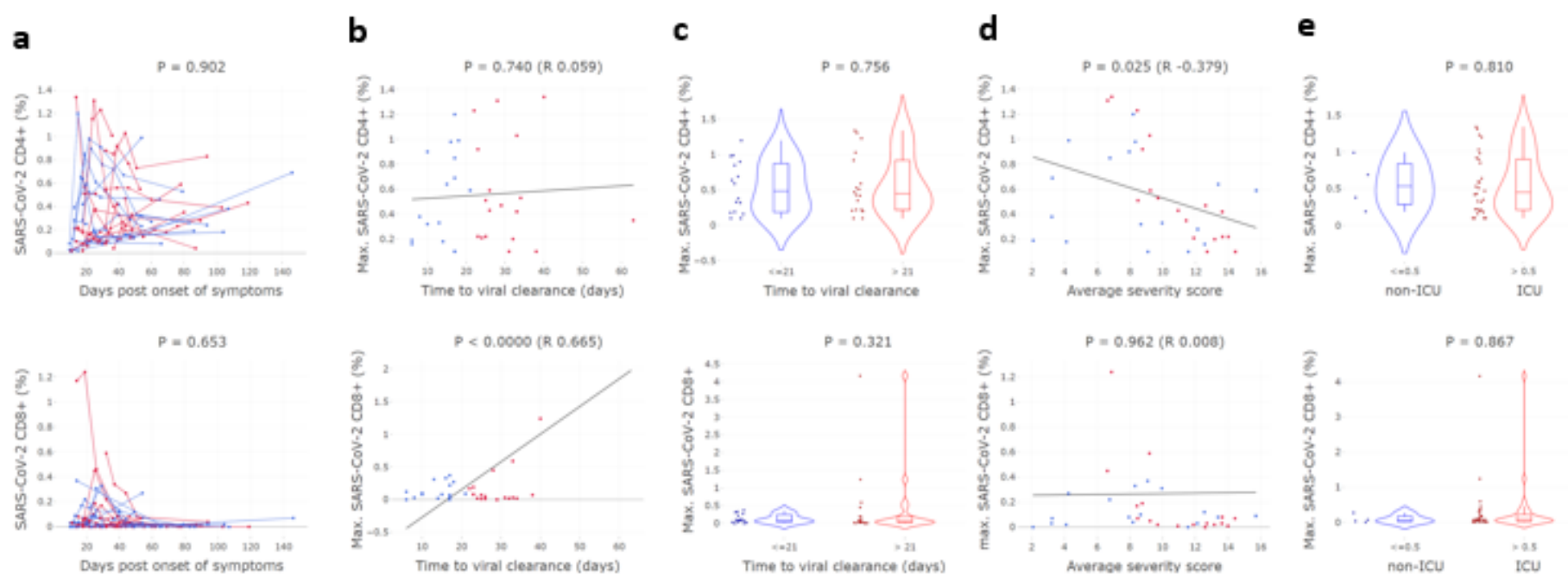

\section{Figure 5}

SARS-CoV-2 specific CD4+ and CD8+ T-cells in relation to the day of viral clearance. a. kinetics of SARSCoV-2 specific CD4+ and CD8+ T-cells in relation to the time post onset of symptoms, per patient. $b$. maximum values correlated with time to viral clearance. c. maximum values grouped by the time to viral clearance: $\leq 21$ (blue)/ $>21$ (red) days; $\mathrm{N}=35$ patients. $\mathrm{d}$. maximum values correlated with severity scores. e. maximum values grouped by non-ICU (blue)/ ICU (red) patients. SARS-CoV-2 CD4+ (\%) = percentage of CD154+CD137+ out of total CD4+ T cells, SARS-CoV-2 CD8+ $(\%)=$ percentage of CD137+IFNg+ out of total CD8+ T cells. 


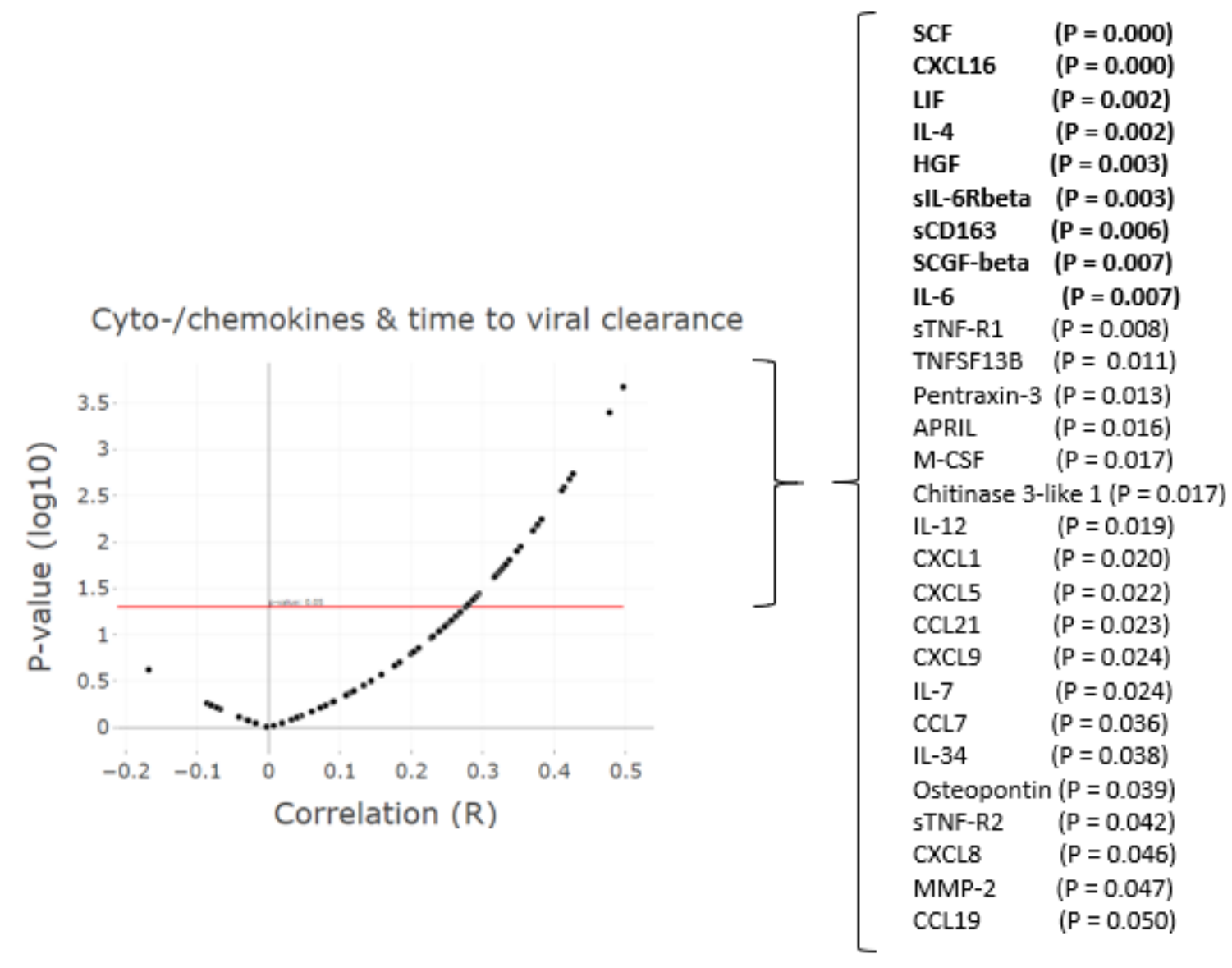

Figure 6

Volcano plot: cytokines and chemokines (maximum levels) correlated with the time to viral clearance, a selection based on P-value $\leq 0.05$, with $R \geq 0.4$ in bold. An overview of all cytokines and chemokines assessed is listed in Extended Data Table 1. 

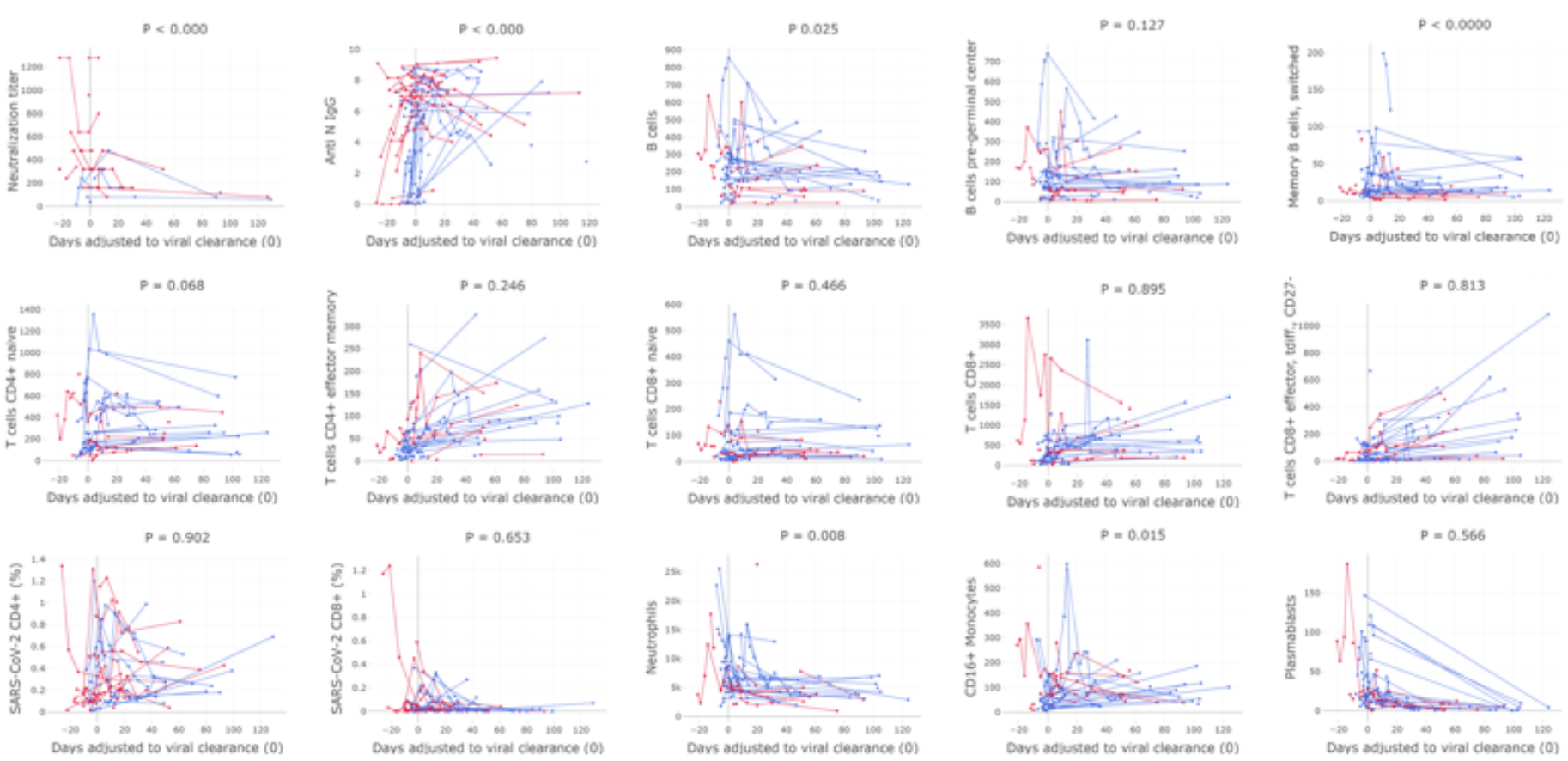

$p=0.015$

$P=0.566$
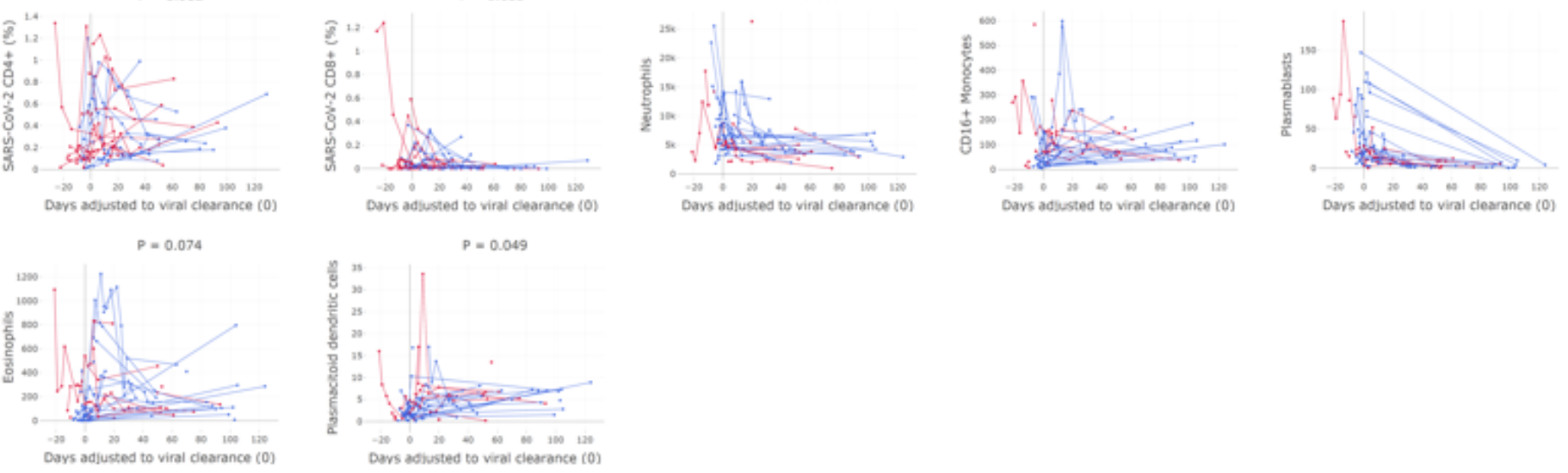

Figure 7

Humoral and cellular immune parameters adjusted to the timing of viral clearance (day 0), indicating peaks of B cells, neutralizing antibodies and SARS-CoV-2 specific CD8+ T cells around viral clearance. A selection of humoral and cellular parameters was made based on findings described above. Blue: viral clearance $\leq 21$ days, red: $>21$ days.
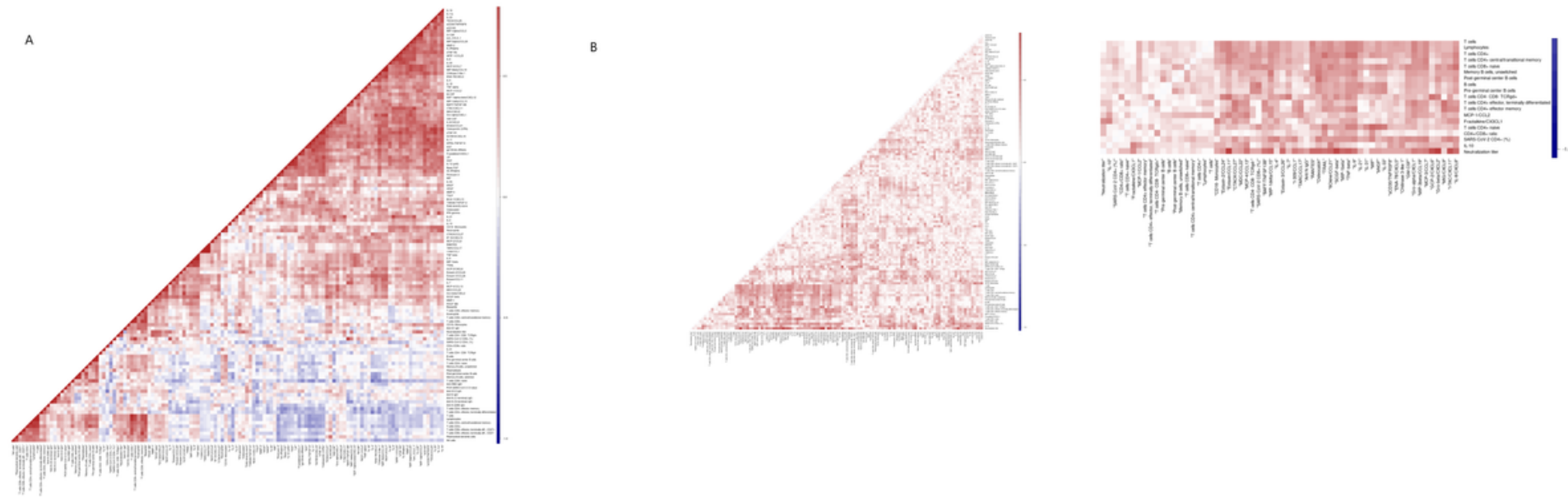

Figure 8 
Correlation heatmaps of the maximum level of immune parameters in individual patients (a), and $b$, for the differences ( $R$ values subtracted) between the patient groups with viral clearance $>21$ days and clearance $\leq 21$ days.

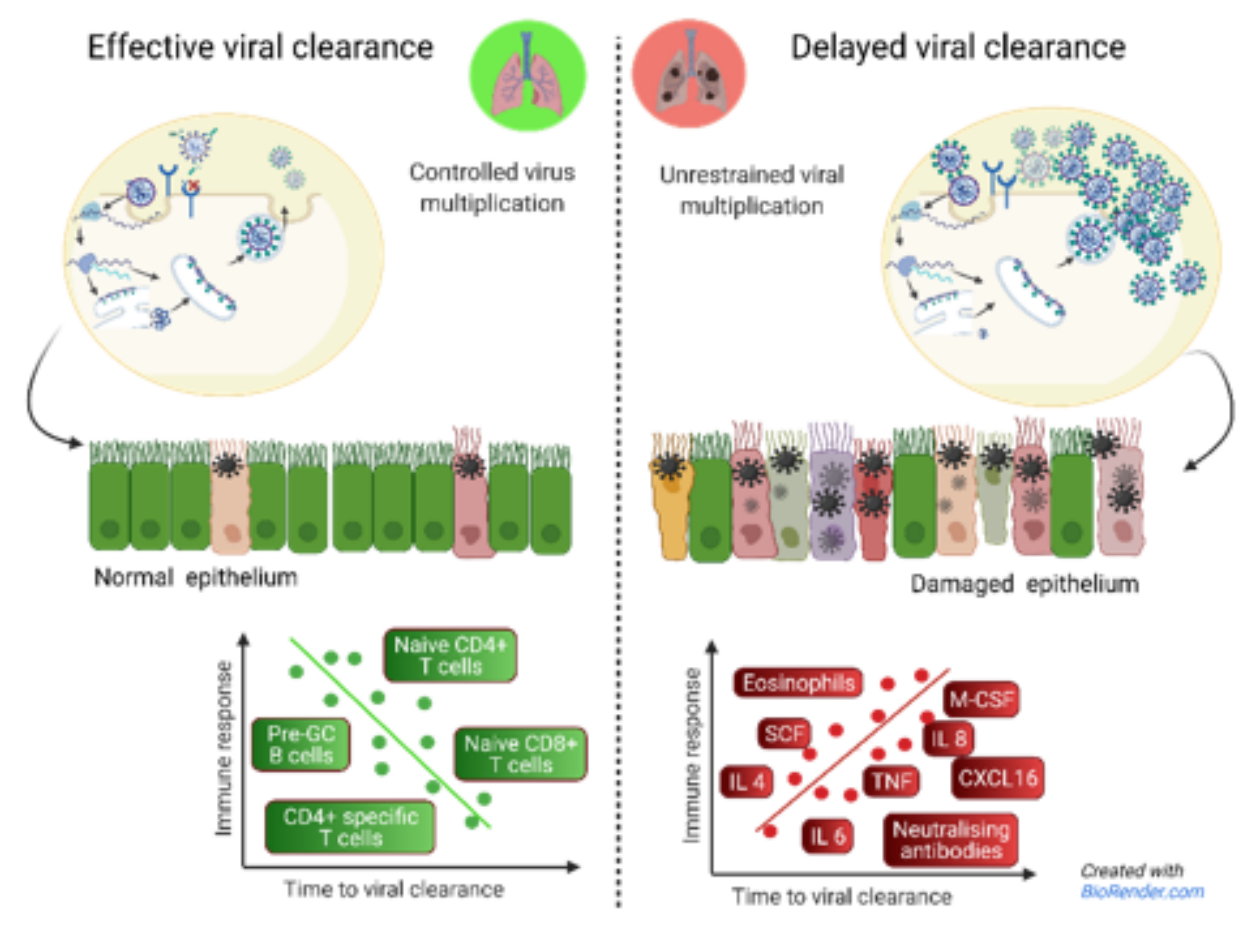

\section{Figure 9}

Hypothesis generating drawing including all immune parameters in this study found to be associated with (delayed) viral clearance. Adapted from "Coronavirus Replication Cycle”, by BioRender.com.

\section{Supplementary Files}

This is a list of supplementary files associated with this preprint. Click to download.

- 03ExtendeddataTables1620210806.xIsx

- 04ExtendeddataFigures20210806.pptx 